\title{
Shared decision-making for prophylactic cranial irradiation in extensive-stage small-cell lung cancer: an exploratory study
}

\author{
Anshu Ankolekar^, Dirk De Ruysscher^, Bart Reymen^, Ruud Houben, Andre Dekker^, \\ Cheryl Roumen^, Rianne Fijten^^
}

Department of Radiation Oncology (MAASTRO), GROW School for Oncology, Maastricht University Medical Center+, Maastricht, The Netherlands

Contributions: (I) Conception and design: A Dekker, C Roumen; (II) Administrative support: A Dekker, C Roumen; (III) Provision of study materials or patients: A Ankolekar, D De Ruysscher, B Reymen, R Houben; (IV) Collection and assembly of data: A Ankolekar; (V) Data analysis and interpretation: A Ankolekar, R Fijten, A Dekker, C Roumen; (VI) Manuscript writing: All authors; (VII) Final approval of manuscript: All authors.

Correspondence to: Rianne Fijten. Paul-Henri Spaaklaan 1, 6229 EN, Maastricht, The Netherlands. Email: rianne.fijten@maastro.nl.

Background: Prophylactic cranial irradiation (PCI) offers extensive-stage small-cell lung cancer (ES-SCLC) patients a lower chance of brain metastasis and slightly longer survival but is associated with a short-term decline in quality of life due to side-effects. This tradeoff between survival and quality of life makes PCI suitable for shared decision-making (SDM), where patients and clinicians make treatment decisions together based on clinical evidence and patient preferences. Despite recent clinical practice guidelines recommending SDM for PCI in ES-SCLC, as well as the heavy disease burden, research into SDM for lung cancer has been scarce. This exploratory study presents patients' experiences of the SDM process and decisional conflict for PCI.

Methods: Radiation oncologists $(n=7)$ trained in SDM applied it in making the PCI decision with ESSCLC patients $(n=25)$. We measured patients' preferred level of participation (Control Preferences Scale), the level of SDM according to both groups (SDM-Q-9 and SDM-Q-Doc), and patients' decisional conflict [decisional conflict scale (DCS)].

Results: Seventy-nine percent of patients preferred a collaborative role in decision-making, and median SDM scores given by patients and clinicians were 80 (IQR: 75.6-91.1) and 85.2 (IQR: 78.7-88.9) respectively, indicating satisfaction with the process. However, patients experienced considerable decisional conflict. Over 50\% lacked clarity about which choice was suitable for them and were unsure what to choose. Sixty-four percent felt they did not know enough about the harms and benefits of PCI, and $60 \%$ felt unable to judge the importance of the harms/benefits in their life.

Conclusions: ES-SCLC patients prefer to be involved in their treatment choice for PCI but a substantial portion experiences decisional conflict. Better information provision and values clarification may support patients in making a choice that reflects their preferences.

Keywords: Shared decision-making (SDM); small-cell lung cancer (SCLC); prophylactic cranial irradiation (PCI); patient-centered; decisional conflict

Submitted Mar 03, 2021. Accepted for publication May 25, 2021.

doi: $10.21037 /$ tlcr-21-175

View this article at: https://dx.doi.org/10.21037/tlcr-21-175

^ORCID: Anshu Ankolekar, 0000-0001-7681-1254; Dirk De Ruysscher, 0000-0002-1214-3557; Bart Reymen, 0000-0003-1709-8414; Andre Dekker, 0000-0002-0422-7996; Cheryl Roumen, 0000-0002-2941-4632; Rianne Fijten, 0000-0002-1964-6317. 


\section{Introduction}

Lung cancer is the leading cause of cancer-related deaths worldwide (1). Small-cell lung cancer (SCLC) accounts for $15 \%$ of lung cancer cases and has a high mortality rate due to rapid progression and metastases (2). Prophylactic cranial irradiation (PCI) offers SCLC patients with distant metastases (so-called "extensive disease" patients) a lower chance of developing brain metastasis, a slightly longer overall survival (OS) (median OS from 5.4 to 6.7 months; 1-year survival rate $27.1 \%$ in the PCI and $13.3 \%$ in the control group), and no long term statistically significant detrimental effect on global health status up to 9 months after treatment (3). In the short term however, PCI is associated with a decline in quality of life as a result of the treatment, mostly due to fatigue. Recent ESMO clinical practice guidelines call for a shared decision-making (SDM) approach to PCI for ES-SCLC patients (4). SDM is an interactive process in which patients and clinicians collaborate in choosing treatments based upon the best available evidence and the patient's personal preferences (5).

Treatment decisions such as whether or not to perform PCI are typically made by clinicians from various specializations in a multidisciplinary tumor board, whose recommendation is then communicated to the patient via the treating clinician. In practice, few patients deviate from this recommendation. This is also the case for extensive stage SCLC patients (ES-SCLC), where PCI is considered a standard treatment for patients with adequate performance status after responding to chemotherapy (6). However, the trade-off between survival and quality of life implies that PCI may be a less attractive option for patients who are expected to have a shorter survival time. Therefore there is a need to take the patient perspective into account (7).

Till date, the SDM process in ES-SCLC has not been explored as the literature tends to focus on preferencesensitive cancer types, such as prostate cancer and breast cancer, while lung cancer decisions tend to be driven by clinical guidelines. As a result, despite the aggressive nature of SCLC and indications of a heavy disease burden in this less studied patient population (8), little attention has been paid to how to include patients and their values and preferences in this complex decision. Patients who engage in SDM experience less decisional conflict, improved compliance with treatment, and greater quality of life compared to usual care in various disease areas (9). Therefore, exploratory work is needed to understand patients' participation desire, experiences, and challenges in the ES-
SCLC decision-making process. A better understanding of the patient perspective in the PCI decision may pave the way for adapting the SDM process to this complex context and developing suitable decision support tools.

The purpose of this exploratory study is to determine the extent to which ES-SCLC patients wish to be involved in the PCI decision, the perceived level of SDM when patients and clinicians take this decision together in an SDM process, and the level and sources of decisional conflict these patients experience.

We present the following article in accordance with the MDAR reporting checklist (available at https://dx.doi. org/10.21037/tlcr-21-175).

\section{Methods}

\section{Participants}

All ES-SCLC patients referred to our clinic (MAASTRO) for PCI who met the following inclusion criteria were invited to participate: (I) WHO performance status 0-2; (II) no history of prior chest radiotherapy; (III) no other active malignancy; (IV) no involvement in another trial which may limit their choice of treatments.

In addition, seven radiation oncologists specialized in lung cancer at MAASTRO took part in this study. These clinicians participated in a one-day workshop covering the fundamentals of SDM theory and practice, specific components of the SDM process, and how to facilitate dialogue with patients at each step. The workshop was designed in collaboration with the Psychology Department of the Elisabeth-TweeSteden Hospital (ETZ Tilburg), based on prior experiences with patient focus groups and healthcare professionals. Led by two researchers specialized in SDM, the format of the workshop was participatory; interaction and inputs from the clinicians were encouraged, and these inputs were used to design supplementary materials such as scripts and infographics to facilitate the SDM steps.

\section{Ethics approval}

This study was conducted in accordance with the Declaration of Helsinki (as revised in 2013). The study was approved by the Internal Review Board of MAASTRO (IRB No. P0148) and the Academic Hospital Maastricht/ Maastricht University's Medical Ethics Review Committee (METC AZM/UM, code: 068, No. 2017-0143) and 
informed consent was taken from all the patients.

\section{Study procedure}

Patients received an SDM consultation with a trained clinician in order to decide whether or not to undergo PCI. The clinician engaged with the patient in the prespecified steps of the SDM process, namely acknowledging the decision to make, inviting the patient's participation, informing the patient about PCI and its advantages and disadvantages, eliciting the patient's preferences, and seeking to reach a decision jointly if the patient was prepared to make a decision. After the consultation, clinicians filled in a questionnaire assessing the level of SDM and patients were given a set of questionnaires assessing a variety of SDM measures. Patient questionnaires were administered at three time points: baseline (within one day of the SDM talk), 5 weeks after SDM, and 11 weeks after SDM.

\section{Outcome measures}

We assessed the decision-making process using the following questionnaires; control preferences scale, SDM-Q-9/SDM-Q-Doc, and the decisional conflict scale (DCS). In addition, patient-reported outcome measures (PROMs) were collected as part of standard procedure (Appendix 1). Validated Dutch translations of all questionnaires were used.

\section{Control preferences scale (CPS)}

The single-item Control Preferences Scale asks respondents to indicate their desired level of participation in a medical decision by selecting one of five statements (10). The statements range from fully active ("I prefer to make the final treatment decision") to fully passive ("I prefer to leave all treatment decisions to my doctor"), with the mid-point being a collaborative role ("I prefer that my doctor and I share responsibility for deciding which treatment is the best").

\section{SDM-Q-9 (patients) and SDM-Q-Doc (clinicians)}

The SDM-Q-9 and SDM-Q-Doc instruments measure patients' and clinicians' assessment of the level of patient involvement in the decision-making process (11). The instrument consists of nine items covering the steps of the SDM process, which can be rated on a six-point scale from 0 (completely disagree) to 5 (completely agree). Patients' and clinicians' raw scores were summed up and rescaled to be on a 0-100 range to be comparable (12). In this study, the patients received the SDM-Q-9 with a five-point Likert scale, whereas the clinicians received the SDM-QDoc with the original six-point Likert scale. As a result, the patient version has a neutral point ('No opinion'), whereas the clinician version induces the respondent to choose between 'Somewhat disagree' and 'Somewhat agree'. Raw scores have been presented for individual item responses to compare between patients and clinicians.

\section{DCS}

The DCS asks patients to report the level of uncertainty they experienced while making their decision using a fivepoint Likert scale (13). This 16-item scale has five subscales: feeling informed, decisional uncertainty, clear values, support, and quality of decisions. Scoring the DCS results in a scale ranging from 0 (no decisional conflict) to 100 (extremely high decisional conflict). Cut-off points are 0-25 (low decisional conflict, high level of decision certainty), 25-37.5 (moderate level of decisional conflict), and above 37.5 (significant uncertainty about decision).

\section{PROMs}

Standard PROMs were collected at all three time points. The EORTC QLQ-C30 measures quality of life of cancer patients along physical, psychological, and social dimensions (14). Finally, the EORTC QLQ-BN20 measures the quality of life of patients receiving treatment for brain tumors along several dimensions, such as future uncertainty, visual disorder, motor dysfunction, and communication deficit (15).

\section{Statistical analysis}

The data from completed questionnaires was scored according to the respective questionnaire's scoring guide. Data analysis was conducted using IBM SPSS. Initial descriptive analysis revealed that SDM-Q-9, SDM-Q-Doc, DCS scores and quality of life scores were not normally distributed, therefore non-parametric tests were used. Patient and clinician SDM scores were compared using a Mann-Whitney U test. Spearman's correlation coefficients were calculated to determine the relationship between SDM and DCS. Wilcoxon signed rank tests were used to compare DCS scores over time.

\section{Results}

Between December 2017 and December 2019, 31 patients 
Table 1 Demographic and clinical characteristics of patients and clinicians who participated in the study

\begin{tabular}{|c|c|}
\hline Variable & $\mathrm{n}[\%]$ \\
\hline \multicolumn{2}{|l|}{ Patients } \\
\hline Total & 25 \\
\hline Female & $12[48]$ \\
\hline Male & $13[52]$ \\
\hline \multicolumn{2}{|l|}{ Age (years) } \\
\hline $40-49$ & $1[4]$ \\
\hline $50-59$ & $3[12]$ \\
\hline $60-69$ & $10[40]$ \\
\hline $70-79$ & $10[40]$ \\
\hline $80+$ & $1[4]$ \\
\hline \multicolumn{2}{|l|}{$\mathrm{N}$-stage } \\
\hline $\mathrm{cNO}-\mathrm{cN} 2$ & $8[32]$ \\
\hline cN3 & $17[68]$ \\
\hline \multicolumn{2}{|l|}{ M-stage } \\
\hline M1a or M1b solitary & $4[16]$ \\
\hline M1b (other) & $21[84]$ \\
\hline \multicolumn{2}{|c|}{ WHO performance status } \\
\hline 0 & $4[15]$ \\
\hline 1 & 18 [72] \\
\hline 2 & 3 [12] \\
\hline Clinicians & n [\%] \\
\hline Total & 7 \\
\hline Female & $4[57]$ \\
\hline Male & $3[43]$ \\
\hline \multicolumn{2}{|l|}{ Age (years) } \\
\hline 30-39 & $3[43]$ \\
\hline $40-49$ & 2 [29] \\
\hline $50-59$ & $1[14]$ \\
\hline $60-69$ & $1[14]$ \\
\hline \multicolumn{2}{|l|}{ Experience (years) } \\
\hline $0-5$ & $1[14]$ \\
\hline $6-10$ & $2[29]$ \\
\hline $11-15$ & $1[14]$ \\
\hline $16-20$ & $1[14]$ \\
\hline $20+$ & $2[29]$ \\
\hline
\end{tabular}

consented to take part in the study. After the SDM talk, the majority of patients $(84 \%)$ chose to undergo PCI, while five patients $(16 \%)$ chose not to. The median survival time of all patients was 26.6 weeks (SD: 20.2).

Twenty-five patients returned baseline questionnaires, yielding a completion rate of $80.6 \%$. Out of these 25 patients, 18 returned the 5-week follow-up questionnaires and 15 returned the 11-week follow-up questionnaires. Two patients were deceased by the time of their 11-week follow-up but only four patients remained alive at the closing date of the study (May 2020), indicating that disease progression could be a major factor in loss to follow-up.

Characteristics of the 25 baseline patients and the seven participating clinicians are presented in Table 1. The patient sample was roughly evenly split across gender and the mean age was 66.8 years (SD: 8.2). TNM staging was indicative of extensive stage SCLC, with the majority of patients having metastasis to the lymph nodes and distant metastasis. The majority of patients had a performance status of 1 or lower, indicating an ability to carry out light activities; the remaining $12 \%$ of patients had a performance status of 2 indicating ability to perform self-care but not work activities.

\section{Patients' preferred level of involvement in decision-making}

The majority of patients (79\%) preferred to play an active or collaborative role in their treatment decision, as measured by the CPS (Figure 1). Of these, one patient preferred to make the decision alone, while the remaining patients preferred to share the decision-making role with their clinician to varying degrees. Five patients (approximately $20 \%$ ) preferred a more passive role in which the clinician makes the final decision. These findings remained fairly consistent across the three measurement points, indicating that patients' experience of their treatment choice did not change their preferred role in the decision-making process.

\section{The level of SDM perceived by patients and clinicians}

With 100 representing the highest level of SDM, the median SDM score at baseline was 80 (IQR: 75.6-91.1) according to patients and 85.2 (IQR: 78.7-88.9) according to clinicians (Figure 2). There was a higher variability in patient SDM scores, however a Mann-Whitney U test showed no significant difference in median SDM scores between patients and clinicians as a whole (z-score -0.514 , $\mathrm{P}=0.610$ ). Spearman's rho correlation between matching 


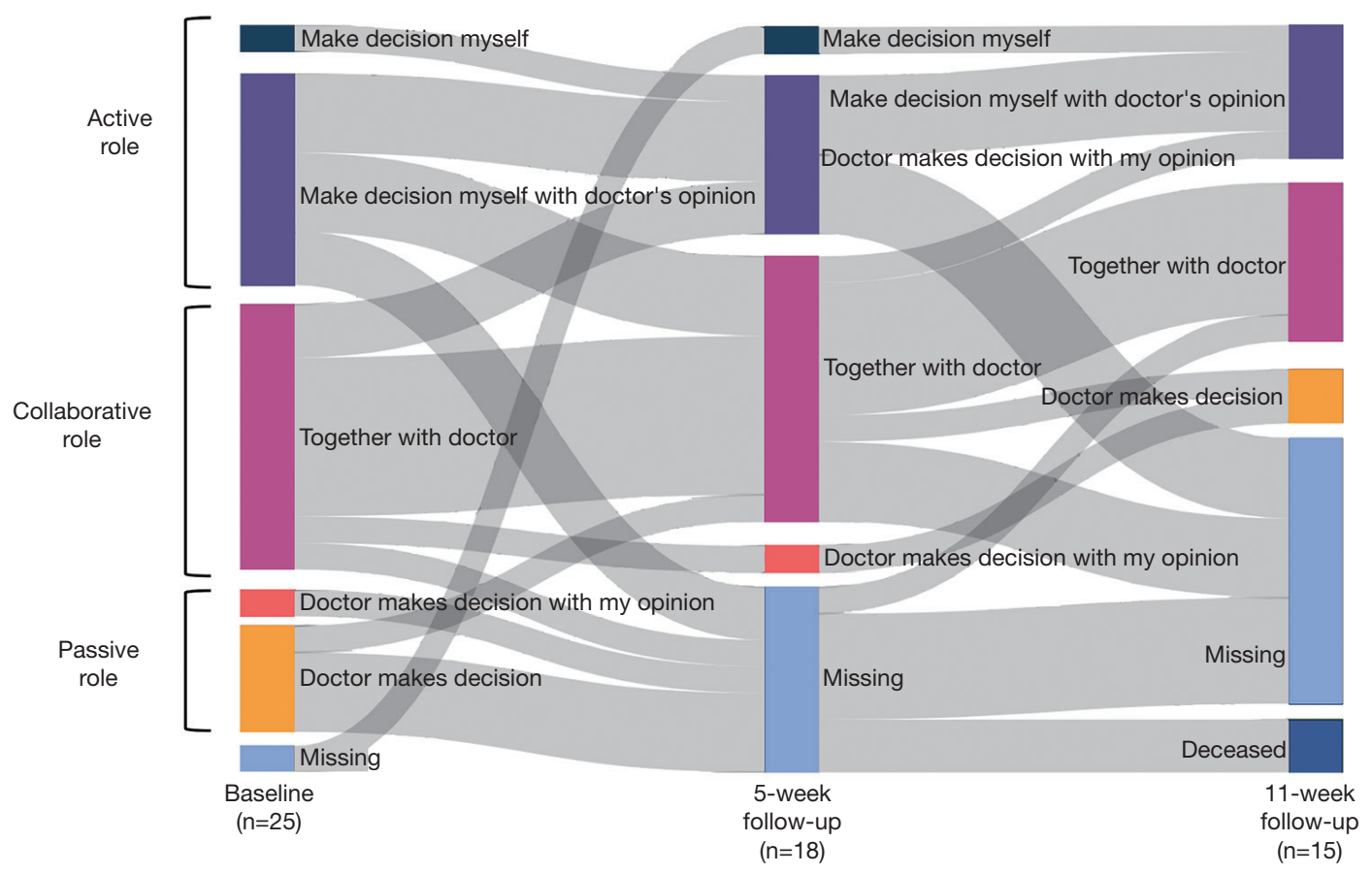

Figure 1 Control Preference Scale measurements showing patients' preferred level of participation in the decision, ranging between active ("I prefer to make the decision myself" or "I prefer to make the decision myself with the doctor's opinion"), collaborative ("I prefer to make the decision together with the doctor"), and passive ("I prefer that the doctor makes the final decision with my opinion considered" or "I prefer that the doctor makes the decision entirely"). Although there was a notable amount of missing data, particularly in the follow-up periods, we observe a tendency towards a collaborative role; patients who preferred an active role at baseline later preferred to include the clinician's opinion or indeed make the decision together with the clinician. Patients who preferred a collaborative role at baseline tended to retain that preference over time or indeed shift their preference to a more collaborative role. A minority of patients preferred a passive role.

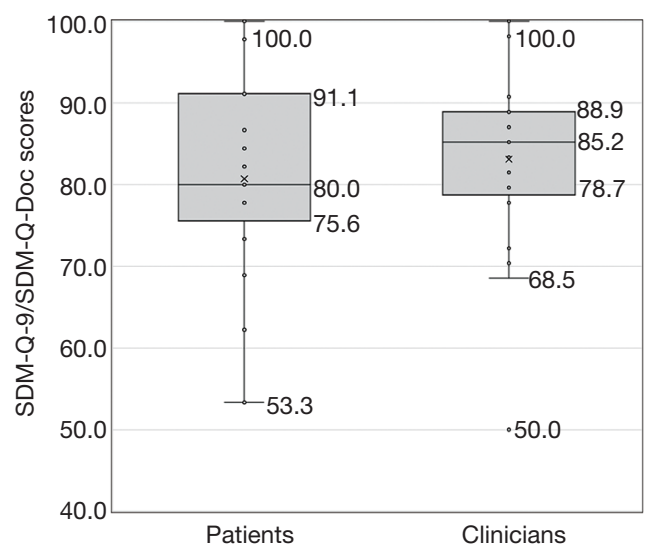

Figure 2 Patients' and clinicians' perceptions of the SDM process at baseline. The vertical axis represents SDM scores rescaled to a $0-100$ scale, with 0 (not shown) indicating no SDM and 100 representing the highest level of SDM. Responses ranged from 50100, with median 80 (patients) and 85.2 (clinicians). SDM, shared decision-making. patient-clinician pairs indicated no significant pairwise correlation $\left(r_{s}=0.287, \mathrm{P}=0.164\right)$.

These results indicate that patients and clinicians did not differ significantly as a group in their estimation of the SDM level, however their pairwise agreement was low. To investigate this further, we compared patient and clinician responses on individual items of the SDM-Q-9 and SDMQ-Doc questionnaire (Figure 3).

Patients and clinicians were in agreement that it was sufficiently clear that a treatment decision needs to be made (Item 1) and that corresponding information were adequately explained (Items 4 and 5). Most patients (84\%) felt that their clinician wanted to know to what extent the patient wishes to be involved in decision-making, and an equal percentage of clinicians reported the same (Item 2).

Patients and clinicians differed on whether the treatment options were presented adequately (Item 3), with more patients disagreeing on this statement. There was also 


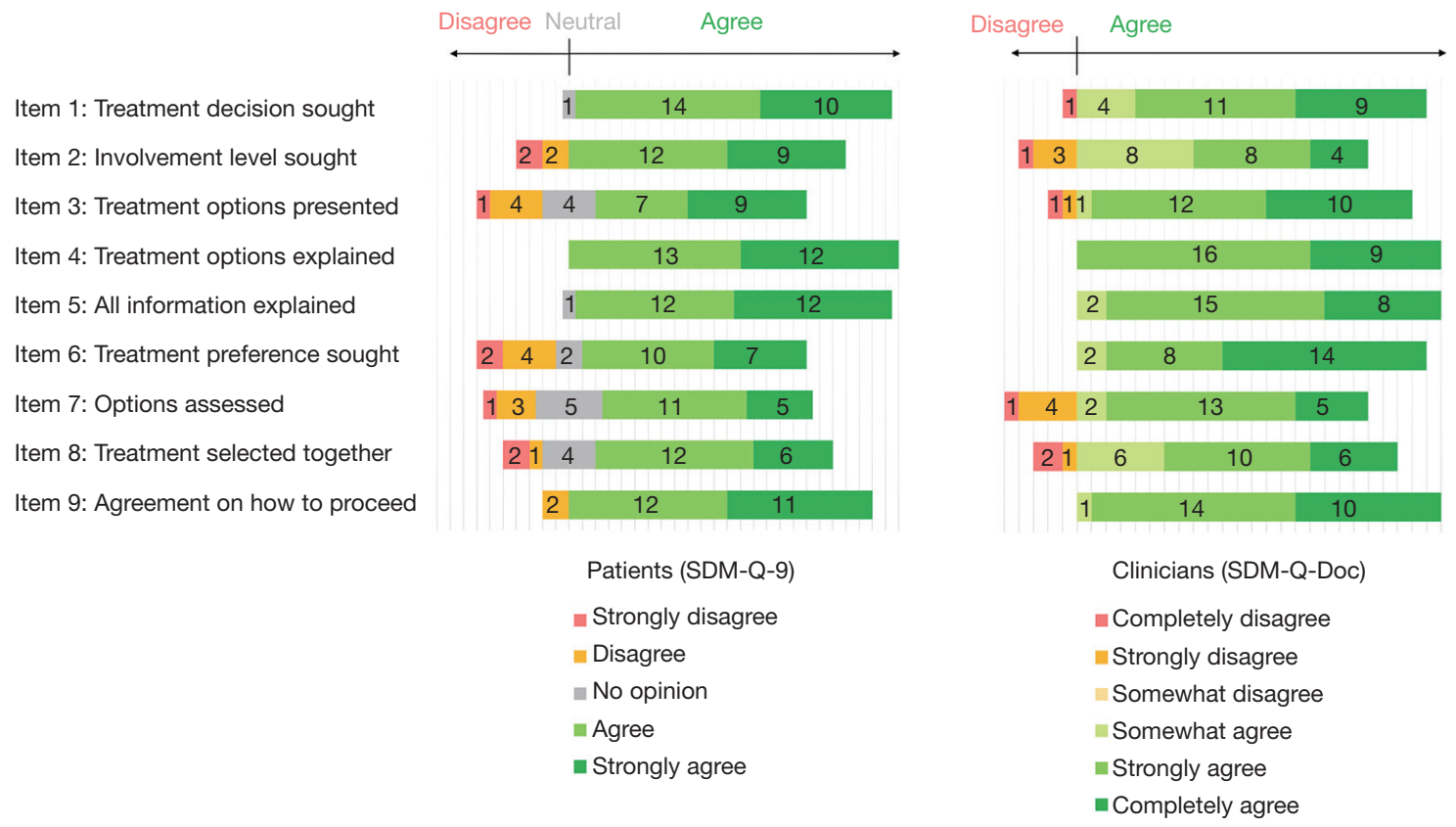

Figure 3 A comparison of the item scores on the SDM-Q-9 questionnaire from 25 patients and the SDM-Q-Doc questionnaire from seven clinicians who participated in the consultations of these 25 patients. The vertical axis displays the individual items in questionnaire and the horizontal axis represents the level of agreement with the items. The scales between patients and clinicians differed, namely patients answered on a five-point Likert scale with a neutral mid-point ('No opinion') whereas clinicians answered on a six-point Likert scale with no neutral mid-point. SDM, shared decision-making.

disagreement on the extent to which the patient's treatment preference was explicitly sought (Item 6); all clinicians agreed to varying degrees that they asked the patient which treatment option he/she prefers, while only $68 \%$ of patients agreed with the same statement. Finally, we observed a discrepancy between patients and clinicians regarding whether the treatment options were thoroughly weighed (Item 7). Here again, patients disagreed with these statements more than clinicians.

Spearman's correlation between patient SDM scores and their CPS scores was positive and statistically significant $\left(r_{s}=0.501, P=0.013\right)$, indicating that patients with higher SDM scores tended to prefer a more shared decision process with their clinician.

\section{The extent of decisional conflict perceived by patients}

The level of decisional conflict patients experienced with making the choice whether to undergo PCI is presented in Figure 4. The median decisional conflict score for patients at baseline was 21.9 (IQR: 3.1-25) out of 100, and nine patients $(36 \%)$ reported experiencing decisional conflict at baseline. The level of decisional conflict rose in the weeks following the decisional talk: five weeks and 11 weeks after baseline the median DCS was 17.2 (IQR: 3.9-32.4) and 25 (IQR: 14.1-28.1) respectively. DCS scores were not significantly correlated with patients' age $\left(r_{s}=0.086\right.$, $\mathrm{P}=0.681$ ). Wilcoxon signed rank tests showed no significant difference in median paired DCS scores from baseline to 5-week follow-up and from 5 -week to 11-week follow-up.

The three most significant sources of decisional conflict for patients were uncertainty, not feeling sufficiently informed, and a lack of clarity about their own personal values (Figure 5). Patients experienced the most severe decisional conflict on the uncertainty subscale; over $50 \%$ reported a lack of clarity about which choice is best, feeling unsure about what to choose, and that the decision was difficult to make. For $20 \%$ of patients this feeling was pronounced enough for them to feel that they might not have made the right decision. Most patients (64\%) also experienced moderate to severe decisional conflict on the informed subscale, indicating a self-reported lack of awareness about the potential benefits and risks of PCI. Subsequently, $60 \%$ of patients also reported not being able 


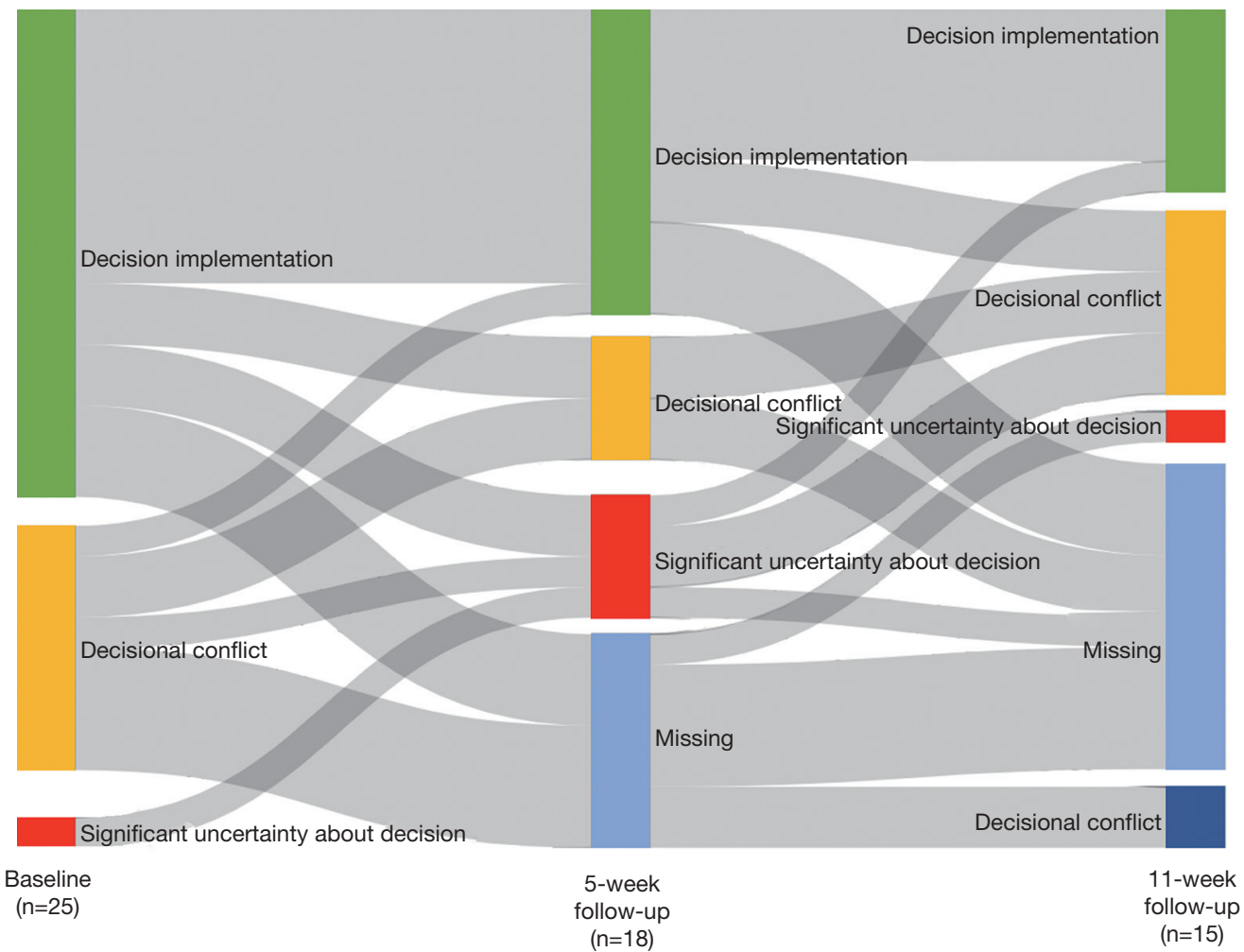

Figure 4 Patients' decisional conflict levels measured by the Decisional Conflict Scale at baseline and 5-week and 11-week follow-ups 'Decision implementation' (green bar) indicates that patients experienced little to no conflict in making their choice. 'Decisional conflict' (yellow bar) refers to a moderate level of decisional conflict, and 'Significant uncertainty about decision' (red bar) indicates severe difficulty in making the treatment decision and doubt whether the best decision was made. Missing data and deceased patients are indicated by light blue and dark blue bars respectively.

to judge how important the benefits and harms would be to them, as indicated by the values clarity subscale.

Patients' perceived level of SDM was significantly inversely correlated with their level of decisional conflict $\left(r_{s}=-0.507, P=0.01\right)$. In other words, patients who rated the level of SDM as higher tended to report having lower levels of decisional conflict. There was no statistically significant correlation between clinicians' SDM scores and patients' decisional conflict $\left(\mathrm{r}_{\mathrm{s}}=0.106, \mathrm{P}=0.614\right)$.

\section{Discussion}

PCI is currently given as a standard treatment to ESSCLC patients as a way to limit the development of brain metastases. However, it can lead to severe short-term sideeffects such as cognitive decline and fatigue $(16,17)$. This raises the question of whether PCI is preferable for patients who may have a poor survival prognosis. This dilemma is exemplified by a median survival of around 6 months in our study population, which is insufficient time to reap the long-term benefits of PCI. The median OS in the present study is similar to that in the EORTC study by Slotman et al. on which the current guidelines are based (3). This, and other recent work, highlights the need to take patient preference into consideration for this decision through an SDM process (17).

In this exploratory study we implemented a simple SDM model and explored the level of SDM and decisional conflict perceived by ES-SCLC patients and their clinicians in making the decision whether to undergo PCI to reduce the risk of brain metastases. A sizable portion of patients in our sample $(16 \%)$ chose not to undergo the guideline-recommended PCI. This deviation is higher than previously observed in our clinic since PCI has been offered as the standard treatment to ES-SCLC patients, however due to a small sample size our study is not sufficiently powered to establish the statistical 


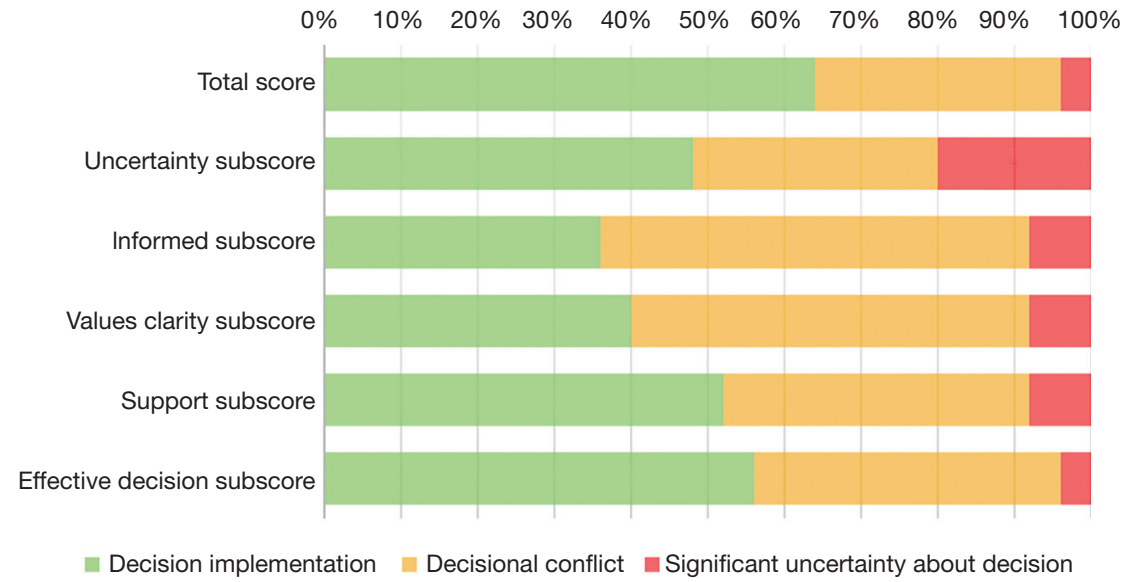

Figure 5 A breakdown of the decisional conflict subscales at baseline. "Decision implementation" indicates that patients faced little to no decisional conflict. Decision implementation' (green bar) indicates that patients experienced little to no conflict in making their choice. 'Decisional conflict' (yellow bar) refers to a moderate level of decisional conflict, and 'Significant uncertainty about decision' (red bar) indicates severe difficulty in making the treatment decision.

significance of this finding. Nevertheless, our study offers insights into patients' experience of the decision-making process and how it may be improved.

\section{Patient participation and preferences for SDM}

We found that patients and clinicians rated the level of SDM highly, though there was no significant pairwise correlation between patient SDM scores and clinician SDM scores. Indeed, we observed discrepancies between patients and clinicians, for instance, while all the clinicians agreed to varying degrees that they had asked their patient's preference, a sizable portion of patients disagreed. Such discrepancies have been observed in prior research through independent observation using the OPTION scale, a validated 12 -item instrument that measures the extent to which a clinician involves a patient in the decision-making process (18). These findings reveal that although many clinicians may feel that they practice SDM, the actual level of SDM might not be as high as they perceived (19). This may be due to varying definitions of what constitutes SDM, for instance, some clinicians conflate it with informed decision-making (20). Our results and these previous findings suggest that when designing SDM training, certain aspects of the SDM process may require more attention and emphasis. In particular, active engagement through role-play may help clarify the difference between informed decision-making and SDM (21). Evaluation of SDM implementation could also include the OPTION scale as an objective measure of the effectiveness of SDM training.

The majority of patients in our sample preferred a collaborative role in the decision-making process, with only a small portion preferring a completely passive role in which the clinician chooses the treatment and even fewer preferring a completely active role with no input from the clinician. This confirms prior research that finds a similar distribution of participation preference among lung cancer patients $(22,23)$. We also found a correlation between CPS and SDM, suggesting that patients who preferred a more active role were more ready to engage in the SDM process and consequently gave a higher score on the SDM-Q9. An alternative interpretation could be that having undergone an SDM process, patients found that they would prefer to be involved in their medical decisions in general, and their CPS scores reflected this. In either case, the discrepancies observed between patient and clinician SDM scores suggest that while clinicians are successfully able to engage patients in making choices, more effort may be needed to help them discover how their treatment choices may impact their quality of life. In other words, the clinician's role should not be to give patients all the relevant information and let them decide, but to take an active role in supporting the patient for truly shared decisions.

\section{Decisional conflict levels}

We found that $36 \%$ of patients experienced decisional conflict when deciding whether to undergo PCI, mainly due 
to the feeling of not understanding the benefits, risks, or sideeffects of PCI, and lack of clarity about which option was best for them. Similar findings have been reported in the Dutch and Korean contexts $(22,24)$. For instance, the former study finds that $40 \%$ of early-stage NSCLC patients experience decisional conflict, mostly with respect to the informed and uncertainty subscales. In lung cancer, it seems that treatment options and outcomes are often hard to grasp for nonexperts and that there often are no treatment options with desired outcomes, especially given the stage of the disease that we studied. Future work should find ways to inform patients better, but it is likely these patients will continue to experience a high level of decisional conflict until better treatment options become available for this disease.

Our finding of a significant correlation between SDM and DCS scores suggests that when patients feel involved in the decision-making process, they experience lower decisional conflict. This relationship conforms with similar studies $(25,26)$. Taken together, these findings underline the importance of SDM for the PCI decision and suggest that the SDM process should place significant emphasis on deliberating on the risks and benefits of the treatment options and what they may imply for individual patients. Prior research has shown that access to accurate clinical information improves people's ability to manage decisional uncertainty (27).

\section{Strengths and limitations}

It is important to bear in mind some limitations of our study design. First, the sample size of our study (25 patients) limits the generalizability of our results. The ES-SCLC patient group is a small sub-population of lung cancer patients and recruiting a sufficiently powered sample is a challenge. Previous landmark studies on PCI for ESSCLC patients have required a large number of centers in order to obtain sufficiently powered samples $(28,29)$. Our ability to extend across multiple centers was limited by our goal of implementing SDM training, as designing and conducting such trainings requires considerable resources and coordination. Challenges in data collection were exacerbated by the rapid progression of the disease, which was a possible reason for the missing data in our sample; out of 25 patients, seven were lost to follow-up by the 5 -week mark and 12 by the 11-week mark, of which two patients were deceased. This affects the strength of our conclusions, particularly where statistical significance could not be established. On a related note, a second limitation is that we were not able to include a control group of ESSCLC patients who did not undergo an SDM talk for PCI due to the limited number of patients treated in this clinical use-case. Consequently, we were unable to determine the baseline level of decisional conflict for usual care ES-SCLC patients. Our findings could be strengthened by conducting a multi-center trial to generate a larger sample. In addition, we explore patient and clinician perceptions of the level and process of SDM; we were unable to conduct independent observations of the level of SDM, such as through the OPTION scale. An additional objective measurement may provide deeper insights into the extent of patient participation, particularly since we found discordance between patients' and clinicians' perceptions on certain aspects of the SDM-Q-9/SDM-Q-Doc questionnaires.

Notwithstanding these limitations, this study adds to our understanding of the decision-making process in ESSCLC, a disease area that has not been adequately explored in the context of SDM. Indeed, SCLC has been referred to as an orphan disease due to its low prevalence (30), yet its aggressive nature and high economic burden warrant a deeper exploration of the patient perspective (8). Although our data is limited, our study may function as a starting point for discussion in the medical community about how best to involve patients in this value-laden decision, particularly in light of upcoming ESMO clinical practice guidelines that recommend SDM for the PCI choice for ES-SCLC patients (4). Little is known about patient preferences regarding PCI. Discrete choice experiments for PCI with stage III NSCLC patients suggest that survival benefit is the most important consideration for that patient population (31). Patients were willing to tolerate a significant degree of toxicity if it meant greater survival benefit, and as potential survival benefits decreased so did the level of toxicity the patients were willing to accept. In particular, patients were willing to trade-off potential memory loss and their ability for self-care for a decrease in likelihood of brain metastases.

Future work could use PROMs to explore more extensively the specific dimensions of quality of life that matter to PCI patients in order to guide SDM consultations. For instance, changes in quality of life measures in the weeks following PCI may provide some indication as to which side-effects and toxicities decline most severely. This may assist clinicians in focusing the SDM talk and provide a basis for asking patients about what matters to them. Future research could also focus on developing clinical decision support tools which present individualized risk predictions 
for PCI based on PROMs (32). This may be useful in light of our findings and those of NSCLC in the curative setting (22) that show that one of the biggest sources of decisional conflict is patients' difficulties in balancing the harms and benefits of their treatment options.

To conclude, the added value of PCI for ES-SCLC patients is still being debated in the clinical community (33). This study shows that patients who are engaged in SDM on whether to undergo PCI feel sufficiently involved in the decision-making process, but a considerable portion still experience decisional conflict, particularly in understanding the benefits and risks of the treatment. This leads to feelings of uncertainty about whether they made the best choice. While it may not be possible to eliminate decisional conflict entirely, clinical tools such as prognostic models may provide a clearer picture of the added benefit of PCI in the individual patient. Such tools must be combined with SDM with an emphasis on risk communication so that ES-SCLC patients can make a choice based on their preferences and the best available evidence.

\section{Acknowledgments}

We would like to thank Annick van Manen (ETZ Tilburg) for her contribution in conducting the SDM workshop, Jean Coenen (MAASTRO) for developing supporting SDM materials, and Sylvie Canisius (MAASTRO) for her assistance regarding the study design and data collection. We would also like to thank all the patients and clinicians who participated in this study.

Funding: This study was supported by CZ, a Dutch health insurance company, as part of a program titled 'My Best Treatment' aimed at improving personalized patientcentered care in the Netherlands.

\section{Footnote}

Reporting Checklist: The authors have completed the MDAR reporting checklist. Available at https://dx.doi. org/10.21037/tlcr-21-175

Data Sharing Statement: available at https://dx.doi. org/10.21037/tlcr-21-175

Peer Review File: Available at https://dx.doi.org/10.21037/ tlcr-21-175

Conflicts of Interest: All authors have completed the ICMJE uniform disclosure form (available at https://dx.doi. org/10.21037/tlcr-21-175). Dr. RF reports that this project was funded by $\mathrm{CZ}$, a health insurance company. In addition, Dr. RF has previously received funding from Varian Medical Systems for another unrelated project. The authors have no other conflicts of interest to declare.

Ethical Statement: The authors are accountable for all aspects of the work in ensuring that questions related to the accuracy or integrity of any part of the work are appropriately investigated and resolved. This study was conducted in accordance with the Declaration of Helsinki (as revised in 2013). The study was approved by the Internal Review Board of MAASTRO (IRB No. P0148) and the Academic Hospital Maastricht/Maastricht University's Medical Ethics Review Committee (METC AZM/UM, code: 068, No. 2017-0143) and informed consent was taken from all the patients.

Open Access Statement: This is an Open Access article distributed in accordance with the Creative Commons Attribution-NonCommercial-NoDerivs 4.0 International License (CC BY-NC-ND 4.0), which permits the noncommercial replication and distribution of the article with the strict proviso that no changes or edits are made and the original work is properly cited (including links to both the formal publication through the relevant DOI and the license). See: https://creativecommons.org/licenses/by-nc-nd/4.0/.

\section{References}

1. Torre LA, Siegel RL, Jemal A. Lung Cancer Statistics. Adv Exp Med Biol 2016;893:1-19.

2. Huber RM, Tufman A. Update on small cell lung cancer management. Breathe 2012;8:314-30.

3. Slotman BJ, Mauer ME, Bottomley A, et al. Prophylactic cranial irradiation in extensive disease small-cell lung cancer: short-term health-related quality of life and patient reported symptoms: results of an international Phase III randomized controlled trial by the EORTC Radiation Oncology and Lung Cancer Groups. J Clin Oncol 2009;27:78-84.

4. Dingemans A-MC, Früh M, Ardizzoni A, et al. Smallcell lung cancer: ESMO Clinical Practice Guidelines for diagnosis, treatment and follow-up. Ann. Oncol 2021;S0923-7534(21)01113-3.

5. Elwyn G, Frosch D, Thomson R, et al. Shared decision making: a model for clinical practice. J Gen Intern Med 
2012;27:1361-7.

6. Vansteenkiste J, De Ruysscher D, Eberhardt WE, et al. Early and locally advanced non-small-cell lung cancer (NSCLC): ESMO Clinical Practice Guidelines for diagnosis, treatment and follow-up. Ann Oncol 2013;24 Suppl 6:vi89-98.

7. Johnson SB, Decker RH. Prophylactic Cranial Irradiation Versus Surveillance: Physician Bias and Patient-centered Decision-making. Clin Lung Cancer 2018;19:464-6.

8. Enstone A, Greaney M, Povsic M, et al. The Economic Burden of Small Cell Lung Cancer: A Systematic Review of the Literature. Pharmacoecon Open 2018;2:125-39.

9. Stacey D, Légaré F, Lewis K, et al. Decision aids for people facing health treatment or screening decisions. Cochrane Database Syst Rev 2017;4:CD001431.

10. Degner LF, Sloan JA, Venkatesh P. The Control Preferences Scale. Can J Nurs Res 1997;29:21-43.

11. Kriston L, Scholl I, Hölzel L, et al. The 9-item Shared Decision Making Questionnaire (SDM-Q-9). Development and psychometric properties in a primary care sample. Patient Educ Couns 2010;80:94-9.

12. Rodenburg-Vandenbussche S, Pieterse AH, Kroonenberg PM, et al. Dutch Translation and Psychometric Testing of the 9-Item Shared Decision Making Questionnaire (SDM-Q-9) and Shared Decision Making Questionnaire-Physician Version (SDMQ-Doc) in Primary and Secondary Care. PLoS One 2015;10:e0132158.

13. O'Connor AM. Validation of a decisional conflict scale. Med Decis Making 1995;15:25-30.

14. Fayers P, Bottomley A; EORTC Quality of Life Group, et al. Quality of life research within the EORTC-the EORTC QLQ-C30. European Organisation for Research and Treatment of Cancer. Eur J Cancer 2002;38 Suppl 4:S125-33.

15. Taphoorn MJ, Claassens L, Aaronson NK, et al. An international validation study of the EORTC brain cancer module (EORTC QLQ-BN20) for assessing health-related quality of life and symptoms in brain cancer patients. Eur J Cancer 2010;46:1033-40.

16. Witlox WJA, Ramaekers BLT, Joore MA, et al. Healthrelated quality of life after prophylactic cranial irradiation for stage III non-small cell lung cancer patients: Results from the NVALT-11/DLCRG-02 phase III study. Radiother Oncol 2020;144:65-71.

17. Putora PM, Glatzer M, Belderbos J, et al. Prophylactic cranial irradiation in stage IV small cell lung cancer: Selection of patients amongst European IASLC and ESTRO experts. Radiother Oncol 2019;133:163-6.

18. Elwyn G, Edwards A, Wensing M, et al. Shared decision making: developing the OPTION scale for measuring patient involvement. Qual Saf Health Care 2003;12:93-9.

19. Couët N, Desroches S, Robitaille H, et al. Assessments of the extent to which health-care providers involve patients in decision making: a systematic review of studies using the OPTION instrument. Health Expect 2015;18:542-61.

20. Moumjid N, Gafni A, Brémond A, et al. Shared decision making in the medical encounter: are we all talking about the same thing? Med Decis Making 2007;27:539-46.

21. Forsetlund L, Bjørndal A, Rashidian A, et al. Continuing education meetings and workshops: effects on professional practice and health care outcomes. Cochrane Database Syst Rev 2009;CD003030.

22. Mokhles S, Nuyttens JJME, de Mol M, et al. Treatment selection of early stage non-small cell lung cancer: the role of the patient in clinical decision making. BMC Cancer 2018;18:79.

23. Hotta K, Kiura K, Takigawa N, et al. Desire for information and involvement in treatment decisions: lung cancer patients' preferences and their physicians' perceptions: results from Okayama Lung Cancer Study Group Trial 0705. J Thorac Oncol 2010;5:1668-72.

24. Sim JA, Shin JS, Park SM, et al. Association between information provision and decisional conflict in cancer patients. Ann Oncol 2015;26:1974-80.

25. Graham ME, Westerberg BD, Lea J, et al. Shared decision making and decisional conflict in the Management of Vestibular Schwannoma: a prospective cohort study. J Otolaryngol Head Neck Surg 2018;47:52.

26. Chorney J, Haworth R, Graham ME, et al. Understanding shared decision making in pediatric otolaryngology. Otolaryngol Head Neck Surg 2015;152:941-7.

27. Rains SA. Health information seeking and the World Wide Web: an uncertainty management perspective. J Health Commun 2014;19:1296-307.

28. Slotman B, Faivre-Finn C, Kramer G, et al. Prophylactic cranial irradiation in extensive small-cell lung cancer. $\mathrm{N}$ Engl J Med 2007;357:664-72.

29. Takahashi T, Yamanaka T, Seto T, et al. Prophylactic cranial irradiation versus observation in patients with extensive-disease small-cell lung cancer: a multicentre, randomised, open-label, phase 3 trial. Lancet Oncol 2017;18:663-71.

30. Oronsky B, Reid TR, Oronsky A, et al. What's New in 
SCLC? A Review. Neoplasia 2017;19:842-7.

31. Lehman M, Gorayski P, Watson S, et al. Patient preferences regarding prophylactic cranial irradiation: A discrete choice experiment. Radiother Oncol 2016;121:225-31.

32. Putora PM, Fischer GF, Früh M, et al. Treatment of brain metastases in small cell lung cancer: Decision-making

Cite this article as: Ankolekar A, De Ruysscher D, Reymen B, Houben R, Dekker A, Roumen C, Fijten R. Shared decisionmaking for prophylactic cranial irradiation in extensive-stage small-cell lung cancer: an exploratory study. Transl Lung Cancer Res 2021;10(7):3120-3131. doi: 10.21037/tlcr-21-175 amongst a multidisciplinary panel of European experts. Radiother Oncol 2020;149:84-8.

33. Picardi C, Caparroti F, Di Maio M, et al. Prophylactic cranial irradiation in extensive disease small cell lung cancer: An endless debate. Crit Rev Oncol Hematol 2019;143:95-101. 


\section{Appendix: Patient-reported Outcome Measures}

Standard patient questionnaires collected as part of patient-reported outcomes (PROMs) included:

EORTC QLQ-C30: A questionnaire developed to assess the quality of life of cancer patients. The QLQ-C30 is composed of both multi-item scales and single-item measures. These include a global health status/QoL scale, five functional scales, three symptom scales, and six single items. All of the scales and single-item measures range in score from 0 to 100 . For global health status and functional scales, a higher score indicates better QoL. For symptom scales, a higher score indicates worse symptoms.

EORTC QLQ-BN20: A specialized questionnaire developed to assess the quality of life of patients undergoing chemotherapy or radiotherapy for brain tumors. It includes 20 items with four multi-item scales (future uncertainty, visual disorder, motor dysfunction, and communication deficit), and single-item symptom scales (e.g. headaches and seizures). When the questionnaire is scored, all scales and items range from 0 to 100, with a higher score representing worse symptoms.

\section{EORTC QLQ-C30}

EORTC QLQ-C30 scores at baseline and follow-up are presented in Table S1. We observe a moderate level of QoL at baseline (median score: 66.7, IQR: 58.3-83.3) that remained relatively constant over follow-up with a slight increase in median score 11 weeks after SDM (median score: 75.0, IQR: 54.2-83.3). On functional scales, patients reported the highest scores on the cognitive and social domains, though the median social functioning score declined in the 5 weeks following SDM from 100 (IQR: 83.3-100) to 66.7 (IQR: 50.0-83.3). Role functioning had the lowest median score throughout the observation period.

Fatigue and dyspnea were the most commonly reported symptoms at baseline, with a median score of 33.3 (IQR: 22.2-44.4 and 0.0-33.3 respectively). Median scores for appetite loss, pain, and nausea rose from 0.0 at baseline to 33.3 (IQR: 0.0-66.7), 25.0 (IQR: 0.0-33.3), and 16.7 (IQR: 0.0-50.0) at the first follow-up, and remained at these levels with the exception of pain which declined slightly to 16.7 (IQR: 0.0-33.3). These values are comparable with European references values for ES-SCLC patients [29].

\section{EORTC-QLQ BN20}

Table $S 2$ shows that of the four multi-item scales, patients reported the highest levels on future uncertainty at baseline, with a median score of 33.3 and IQR 22.2-44. Of the symptom scales, hair loss was the most severe symptom; the median score of 66.7 at baseline rose to 100.0 (IQR: 66.7-100) five weeks after SDM. This may be related to chemotherapy treatments prior to making the PCI decision. Similarly, headaches, drowsiness, and itchy skin were reported to be most severe at the 5-week mark and declined by the 11-week mark. 
Table S1 Patients' median EORTC QLQ-C30 scores at baseline, 5-week follow-up, and 11-week follow-up. Interquartile ranges are reported in parentheses. Scores range from 0-100. For global health status and the five functional scales, a score of 100 indicates the best quality of life. For symptom scales, a score of 100 indicates the worst symptoms.

\begin{tabular}{|c|c|c|c|}
\hline EORTC QLQ-C30 (median, IQR) & Baseline $(n=25)$ & 5-week follow-up $(n=18)$ & 11-week follow-up $(n=15)$ \\
\hline Global health status/QoL & $66.7(58.3-83.3)$ & $66.7(50.0-81.3)$ & $75.0(54.2-83.3)$ \\
\hline \multicolumn{4}{|l|}{ Functional scales } \\
\hline Physical functioning & $75.0(60.0-80.0)$ & $60.0(40.0-73.3)^{\dagger}$ & $73.3(46.7-80.0)$ \\
\hline Role functioning & $66.7(66.7-83.3)$ & $66.7(33.3-66.7)$ & $66.7(50.0-83.3)$ \\
\hline Emotional functioning & $75.0(66.7-91.7)$ & $79.2(66.7-91.7)$ & $83.3(66.7-95.8)$ \\
\hline Cognitive functioning & $100.0(83.3-100.0)$ & $83.3(66.7-95.8)$ & $83.3(66.7-100.0)$ \\
\hline Social functioning & $100.0(83.3-100.0)$ & $66.7(50.0-83.3)$ & $83.3(66.7-100.0)$ \\
\hline \multicolumn{4}{|l|}{ Symptom scales } \\
\hline Fatigue & $33.3(22.2-44.4)$ & $44.4(44.4-88.9)$ & $44.4(33.3-80.6)$ \\
\hline Nausea & $0.0(0.0-0.0)$ & $16.7(0.0-50.0)$ & $16.7(0.0-33.3)$ \\
\hline Pain & $0.0(0.0-33.3)$ & $25.0(0.0-33.3)$ & $16.7(0.0-33.3)$ \\
\hline Dyspnea & $33.3(0.0-33.3)$ & $33.3(33.3-66.7)$ & $33.3(33.3-66.7)$ \\
\hline Insomnia & $0.0(0.0-33.3)$ & $33.3(8.3-66.7)$ & $33.3(0.0-50.0)$ \\
\hline Appetite loss & $0.0(0.0-33.3)$ & $33.3(0.0-66.7)$ & $33.3(0.0-66.7)$ \\
\hline Constipation & $0.0(0.0-0.0)$ & $0.0(0.0-0.0)$ & $0.0(0.0-33.3)$ \\
\hline Diarrhea & $0.0(0.0-0.0)$ & $0.0(0.0-33.3)$ & $0.0(0.0-33.3)$ \\
\hline Financial difficulties & $0.0(0.0-0.0)$ & $0.0(0.0-33.3)$ & $0.0(0.0-16.7)$ \\
\hline
\end{tabular}

${ }^{\dagger}$ Based on 17 observations due to one missing data point.

Table S2 Patients' median EORTC QLQ-BN20 scores at baseline, 5-week follow-up, and 11-week follow-up. Interquartile ranges are reported in parentheses. Scores range from $0-100$, with 100 representing the worst quality of life/symptoms.

\begin{tabular}{|c|c|c|c|}
\hline EORTC QLQ-BN20 (median, IQR) & Baseline( $n=25)$ & 5-week follow-up $(n=18)$ & 11-week follow-up $(n=15)$ \\
\hline \multicolumn{4}{|l|}{ Scales } \\
\hline Future uncertainty & $33.3(22.2-44.4)$ & $38.9(22.2-63.9)$ & $22.2(11.1-44.4)$ \\
\hline Visual disorder & $0.0(0.0-11.1)$ & $16.7(0.0-33.3)$ & $11.1(0.0-22.2)$ \\
\hline Motor dysfunction & $0.0(0.0-11.1)$ & $11.1(0.0-33.3)$ & $11.1(0.0-22.2)$ \\
\hline Communication deficit & $0.0(0.0-0.0)$ & $0.0(0.0-11.1)$ & $0.0(0.0-22.2)$ \\
\hline \multicolumn{4}{|l|}{ Symptom scales } \\
\hline Headaches & $0.0(0.0-0.0)$ & $33.3(8.3-33.3)$ & $0.0(0.0-33.3)$ \\
\hline Seizures & $0.0(0.0-0.0)$ & $0.0(0.0-0.0)$ & $0.0(0.0-0.0)$ \\
\hline Drowsiness & $0.0(0.0-33.3)$ & $33.3(8.3-66.7)$ & $33.3(0.0-50.0)$ \\
\hline Hair loss & $66.7(0.0-100.0)$ & $100.0(66.7-100.0)^{\dagger}$ & $66.7(16.7-100.0)$ \\
\hline Itchy skin & $0.0(0.0-33.3)$ & $33.3(0.0-66.7)^{\dagger}$ & $0.0(0.0-33.3)^{\ddagger}$ \\
\hline Weakness of legs & $0.0(0.0-33.3)$ & $33.3(0.0-33.3)^{\dagger}$ & $0.0(0.0-33.3)$ \\
\hline Bladder control & $0.0(0.0-33.3)$ & $0.0(0.0-33.3)^{\dagger}$ & $0.0(0.0-0.0))$ \\
\hline
\end{tabular}

\footnotetext{
${ }^{\dagger}$ Based on 17 observations due to one missing data point. ${ }^{\ddagger}$ Based on 14 observations due to one missing data point.
} 